\title{
Determination of the Binding Affinity of Virions of Pandemic Influenza A/California/7/2009 (H1N1pdm09) Virus for Fetuin and Mucin by the Competitive and Kinetic Methods
}

\author{
G. S. Onkhonova ${ }^{a, *}$, S. V. Maltsev ${ }^{a}$, and A. B. Ryzhikov ${ }^{a}$ \\ ${ }^{a}$ State Research Center of Virology and Biotechnology VECTOR, Koltsovo, Novosibirsk oblast, 630559 Russia \\ *e-mail:vector@vector.nsc.ru
}

Received December 20, 2017; revised April 29, 2019; accepted April 30, 2019

\begin{abstract}
The affinity of binding of pandemic influenza A/California/7/2009 (H1N1pdm09) virus for fetuin and mucin was studied. Three methods based on the enzyme-linked immunosorbent assay for the quantitative determination of receptor specificity of influenza virus strains were compared. These methods were competitive Dixon plot analysis in direct coordinates, a competitive method using the half-maximal inhibitory concentration $\left(I C_{50}\right)$, and a kinetic method. It has been found that competitive methods significantly distort the result due to unspecific binding of competitive proteins, while the kinetic method prevents such an effect. In the future, it can be widely used to accurately measure the receptor specificity of the influenza virus with $\alpha 2-3$ and $\alpha 2-6$ type receptors.
\end{abstract}

Keywords: receptor specificity, equilibrium dissociation constant, sialic acid

DOI: $10.1134 / \mathrm{S} 0006350919040134$

Influenza is an acute viral contagious disease that circulates around the world and can infect mammals, birds, and humans. As one of the main causes of morbidity and mortality, influenza causes annual epidemics and even pandemics and is a major public health and economic problem. During the life cycle, all virions have an obligatory phase of attachment to the surface of the target cell. The surface of sensitive cells has a receptor that is specific for each virus, and in turn, the virus has a protein that is used for binding to the cellular receptor. In influenza viruses, this protein is hemagglutinin, while glycolipids or glycoproteins, the terminal fragment of which is sialic acid (primarily $\mathrm{N}$-acetylneuraminic acid (Neu5Ac)) are the receptors on the cell surface.

The structural difference in sialic acid residues attached to glycan chains is a key factor in determining the specificity of the binding of different subtypes of the influenza virus to cellular receptors. There are two main chemical forms of sialic acid ( $\alpha 2-3$ and $\alpha 2-6)$; different strains of the influenza virus differ in affinity for them [1]. Strains of avian influenza virus use sialic acids attached to the next sugar residue via the $\alpha 2-3$ bond (Neu5Ac $\alpha 2-3 \mathrm{Gal}$ ) as a receptor [2]. Receptors of this type are mainly found on epithelial cells of the digestive tract of birds. The strains of the

Abbreviations: ELISA, enzyme-linked immunosorbent assay; PBS, phosphate-buffered saline; PBS-T, phosphate-buffered saline supplemented with Tween-20. human influenza virus predominantly attach to sialic acids with an $\alpha 2-6$ bond (Neu5Ac $\alpha 2-6 \mathrm{Gal})$ [3]. Such receptors are essential for epithelial cells of the human upper respiratory tract. At the same time, a small number of $\alpha 2-3$ receptors are present on the ciliary epithelium and epithelial cells of the human lower respiratory tract. For this reason, there is a risk for people to be infected with a highly pathogenic avian influenza virus, and such situations are usually accompanied by complications for humans or even death.

The enzyme-linked immunosorbent assay (ELISA) is one of the methods for assessment of the receptor specificity of influenza viruses. Stationary competitive and kinetic methods are used to assess the results obtained in ELISA. Despite its simplicity and relatively low cost, ELISA has several disadvantages. First, the rather long period of the experiment is characteristic of all types of analysis, which is inconvenient for a large sample. Secondly, it is impossible to compare the values between different strains of the influenza virus using the semi-quantitative ELISA method. The use of quantitative competitive methods is associated with the problem of unspecific binding of competing proteins, which in some cases results in a significant measurement error. Moreover, competitive methods are correct only for certain strictly selected ratios of competing molecules and their dissociation constants. Selection of working ratios and concentrations may be rather time consuming; therefore, 
researchers often ignore this stage. The kinetic ELISA assay tested in this work is devoid of these disadvantages of semi-quantitative and competitive analyses; it is proposed as the best method for measuring the receptor specificity of influenza virus strains.

\section{MATERIALS AND METHODS}

The kinetic method for determining the dissociation constant. The kinetics of the interaction of influenza virus with receptors can be described by the kinetics of the ligand-receptor interaction. In modeling the process of infection, we suggest that a small amount of virus reaches the epithelium of the respiratory tract, which is covered with excess receptors. The equation for the concentration of the formed complex under the conditions of a small ligand concentration (compared to the concentration of receptors), which were used in this study, is as follows [5]:

$$
\begin{gathered}
{[L R]=A\left(1-e^{-k t}\right),} \\
A=\frac{[R]_{0}[L]_{0}}{K_{\mathrm{d}}+[R]_{0}}, \\
k=k_{+}[R]_{0}+k_{-}, \\
K_{\mathrm{d}}=\frac{k_{-}}{k_{+}},
\end{gathered}
$$

where $[L R]$ is the concentration of ligand-receptor complexes; $[R]_{0}$ is the initial concentration of receptors; $[L]_{0}$ is the initial concentration of ligand (antigen); $K_{\mathrm{d}}$ is the equilibrium dissociation constant of ligand-receptor complexes; $k_{+}$is the rate constant of the forward reaction; $k_{-}$is the rate constant of the reverse reaction; and $t$ is time. The $k$ exponent is selected by approximating the experimental dependence of the concentration of ligand-receptor complexes on time using the function (1). If $k$ is determined at two or more receptor concentrations, then after plotting the dependence of the calculated $k$ parameters on the concentration of added receptors, the equation of a line can be obtained using regression methods, which makes it possible to determine $k_{+}$and $k_{-}$, and hence, the $K_{\mathrm{d}}$ equilibrium dissociation constant.

Competitive methods for determining the dissociation constant. In our case, two types of receptors interact with the ligand during competitive binding according to the following scheme [6]:

$$
\left[L_{1} R\right]=\frac{[L]_{0}\left[R_{1}\right]_{0}}{\left[R_{1}\right]_{0}+K_{\mathrm{d}_{1}}\left(1+\frac{\left[R_{2}\right]_{0}}{K_{\mathrm{d}_{2}}}\right)},
$$

where $\left[L_{1} R\right]$ is the concentration of complexes; $[L]_{0}$ is the concentration of ligand; and $\left[R_{1}\right]_{0},\left[R_{2}\right]_{0}, K_{\mathrm{d}_{1}}$, and $K_{\mathrm{d}_{2}}$ are the concentrations and dissociation constants of competing receptors.
In the case of the competitive Dixon plots, the dissociation constant is the abscissa value of the junction point of the curves of displacement of one receptor by the second one at various concentrations of the first receptor. According to theory, the junction point of the extrusion curves is in the negative region and a result of the graphical solution of the system of equations with two unknowns, one of which is the equilibrium dissociation constant.

Another competitive method for measuring the dissociation constant is the method using the halfmaximal inhibitory concentration $\left(I C_{50}\right)$. The equilibrium dissociation constant in this method can be determined as follows:

$$
K_{\mathrm{d}_{2}}=\frac{I C_{50}}{1+\frac{\left[R_{1}\right]_{0}}{K_{\mathrm{d}_{1}}}},
$$

where $I C_{50}$ is the concentration of the second receptor that inhibits the binding of the first receptor by $50 \%$.

In this study, the mucin and fetuin proteins were used as the first receptor (competitor) and the same proteins labeled with biotin were used as the second receptor. Considering that the dissociation constants for labeled protein and competitor are equal, we obtain the following equation:

$$
K_{\mathrm{d}}=I C_{50}-\left[R_{1}\right]_{0} .
$$

The characteristics of the strain used in the study. The viral strain A/California/7/2009 (H1N1pdm09) was selected for this study. The H1N1 subtype caused pandemics in 1918 and 2009; it also caused a epidemic in Russia in 2016. The H1N1pdm09 strain, which became a serious public health problem, was first detected in North America in April 2009. According to the official data, it spread over more than 200 countries and caused more than 18000 deaths worldwide. The pandemic H1N1 virus spreads among people like a regular seasonal influenza virus and has similar symptoms. However, during the severe form of this disease viral pneumonia develops; without treatment the mortality rate is very high. The receptor specificity of binding of the H1N1(pdm2009) strain was studied in $[7,8]$. It was found that it preferentially binds to receptors of the $\alpha 2-6$ type. However, according to the data reported in [9], it is also claimed that this strain can bind to receptors of the $\alpha 2-3$ type but with lower affinity.

Receptor models. The fetuin and mucin proteins were used as receptor models on the surface of sensitive cells. Influenza virus has an affinity for these proteins through interaction with sialic acids. According to the literature data, fetuin contains $\alpha 2-3$ and $\alpha 2-6$ receptors [10], while mucin predominantly contains $\alpha 2-6$ receptors $[11,12]$, although the number and type of sialic acids depend on the species and different mucin types may contain different types of receptors $[13,14]$. The molecular weight of fetuin used in this 
study is $48.4 \mathrm{kDa}$. In contrast to fetuin, mucin differs greatly according to molecular weight. The currently available data indicate that its values are within a very wide range: from 10 to $1000 \mathrm{kDa}$. We also carried out denaturing gel electrophoresis, which showed that the minimal molecular mass of mucin might be considered approximately equal to the mass of fetuin used in the study. As a result, the mass of the mucin used in this work was estimated as the geometric average of the range known from the literature, i.e., $100 \mathrm{kDa}$, which does not contradict the results of electrophoresis.

Enzyme-linked immunosorbent assay. Enzymelinked immunosorbent assay was selected to determine the receptor specificity. The first stage of work was the optimization of the conditions of direct and competitive interaction of the influenza virus with model proteins: fetuin and mucin. After this, kinetic models were selected for analyzing the ELISA results, and subsequent direct measurements of the binding affinity of the selected strain of influenza virus were carried out using three methods: Dixon plots in direct coordinates, a method using the half-maximal inhibitory concentration $\left(I C_{50}\right)$, and the kinetic method.

Purification of influenza virus. Viral allantoic fluid $(100 \mathrm{~mL})$ with inactivated material was filtered through a filter with a pore diameter of $0.22 \mu \mathrm{m}$ and centrifuged using a Sigma $3-30 \mathrm{~K}$ centrifuge $(12150-\mathrm{H}$ rotor) at $20000 \mathrm{rpm}$ for $3 \mathrm{~h}$. The supernatant was decanted and the plaque was dissolved in $0.5 \mathrm{~mL}$ of phosphate-buffered saline (PBS). The obtained concentrate was chromatographically purified by gel filtration on a column with a resin volume of $20 \mathrm{~mL}$ (Sepharose-4B, Pharmacia, Sweden). The column was equilibrated with PBS buffer. The protein concentration in the final solution of viral particles was measured by the Bradford method.

The competitive method using ELISA. The purified virus in PBS $(50 \mu \mathrm{L})$ at a concentration of $10 \mu \mathrm{g} / \mathrm{mL}$ was sorbed onto a standard 96-well microplate for immunological reactions (SPL Life Science, Korea) overnight at room temperature until the antigen was completely dried. The plate was washed with PBS solution supplemented with $0.05 \%$ Tween-20 (PBS-T) using a PW40 microplate washer (Bio-Rad, United States). The plate was filled with biotinylated mucin or fetuin (Sigma-Aldrich, United States) in the solution for serum dilution (Epitek, Novosibirsk, Russia) and serial two-fold dilutions were carried out in the horizontal direction starting with concentrations of $0.2 \mu \mathrm{g} / \mathrm{mL}$ and $10 \mu \mathrm{g} / \mathrm{mL}$, respectively. A dilution procedure for unlabeled mucin or fetuin was also carried out in the vertical direction starting with the concentration of $500 \mu \mathrm{g} / \mathrm{mL}$. The mixture was pipetted in two repetitions: in four columns with the dried virus and four empty control columns. All actions were then performed according to the standard protocol: the plate was washed, filled with the conjugate solution (streptavidin-horseradish polyperoxidase; Biosan,
Novosibirsk, Russia) in PBS-T at a concentration of $1: 10000$, kept at room temperature for $40 \mathrm{~min}$, and washed again. After washing the conjugate out, the plate was filled $(100 \mu \mathrm{L}$ per well $)$ with a mixture $(1: 30)$ of tetramethylbenzidine and a buffer solution for a substrate (Epitek, Novosibirsk, Russia) and incubated in the dark at room temperature for $30 \mathrm{~min}$. Following this, $50 \mu \mathrm{L}$ of the stop solution $\left(5 \% \mathrm{H}_{2} \mathrm{SO}_{4}\right)$ was added per well and the absorbance was measured using a Multiskan EX photometer (Labsystems, Finland) at a wavelength of $450 \mathrm{~nm}$.

The Kinetic method using ELISA. For the experiment, the purified virus $(50 \mu \mathrm{L}$; a concentration of $10 \mu \mathrm{g} / \mathrm{mL}$ according to the total protein) was sorbed onto a microplate overnight at room temperature until the antigen was completely dried. After sorption, the wells were washed with the PBS-T solution. Biotinlabeled mucin and fetuin were diluted in the solution for serum dilution and serial two-fold dilutions were carried out in four wells in the vertical direction, starting with $0.5 \mu \mathrm{g} / \mathrm{mL}$ and $10 \mu \mathrm{g} / \mathrm{mL}$, respectively. Every $10 \mathrm{~min}$, one strip was removed from the plate, washed with a solution of PBS-T, and placed in a refrigerator $\left(4^{\circ} \mathrm{C}\right)$. After $120 \mathrm{~min}$, the plate was washed, filled with a solution of the conjugate in PBS-T at a concentration of $1: 10000$, kept at room temperature for $40 \mathrm{~min}$, and washed once again. The plate was subsequently filled $(100 \mu \mathrm{L}$ per well) with a mixture $(1: 30)$ of tetramethylbenzidine and a buffer solution for the substrate and incubated in the dark at room temperature for $30 \mathrm{~min}$. Stop solution $\left(50 \mu \mathrm{L} ; 5 \% \mathrm{H}_{2} \mathrm{SO}_{4}\right)$ was added to the well to terminate the reaction and the absorbance was measured using a Multiskan EX photometer at a wavelength of $450 \mathrm{~nm}$.

\section{RESULTS}

According to the results of competitive ELISA, the extrusion curves for the A/California/7/2009 (H1N1pdm09) virus strain were obtained (Fig. 1) and the equilibrium dissociation constants were calculated by Dixon plot analysis in direct coordinates and using the half-maximal inhibitory concentration $\left(I C_{50}\right)$. The values of the junction points of the curves and the $I C_{50}$ parameter were determined using the Scilab 4.0 software package.

The experimental points of kinetic ELISA were approximated using function (1) and the Origin 8 software package (Fig. 2) in order to calculate the $k$ coefficients as exponent index at different initial concentrations of fetuin and mucin. The dependences of the calculated $k$ parameters on the initial concentration of the added protein (fetuin or mucin) were further plotted (Fig. 3), and the equilibrium dissociation constants were calculated by regression methods according to formula (4).

Table 1 contains the values of the equilibrium dissociation constants for the selected strain of influenza 


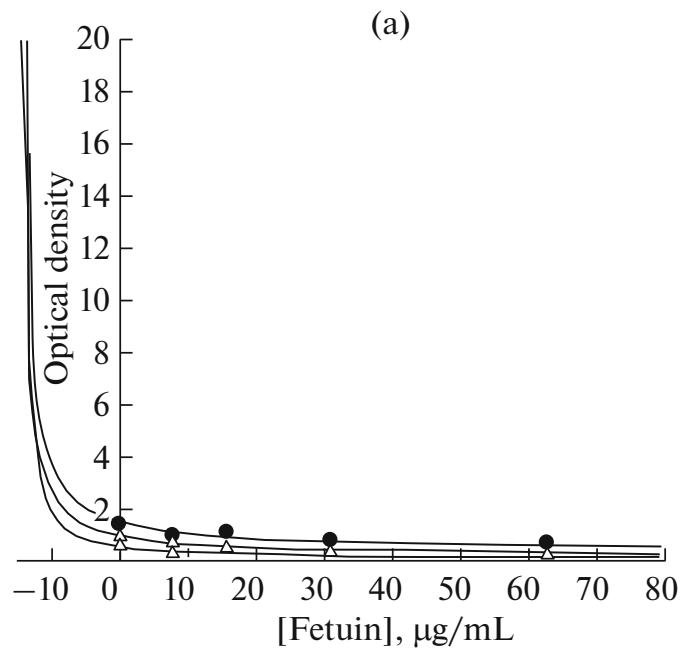

(c)

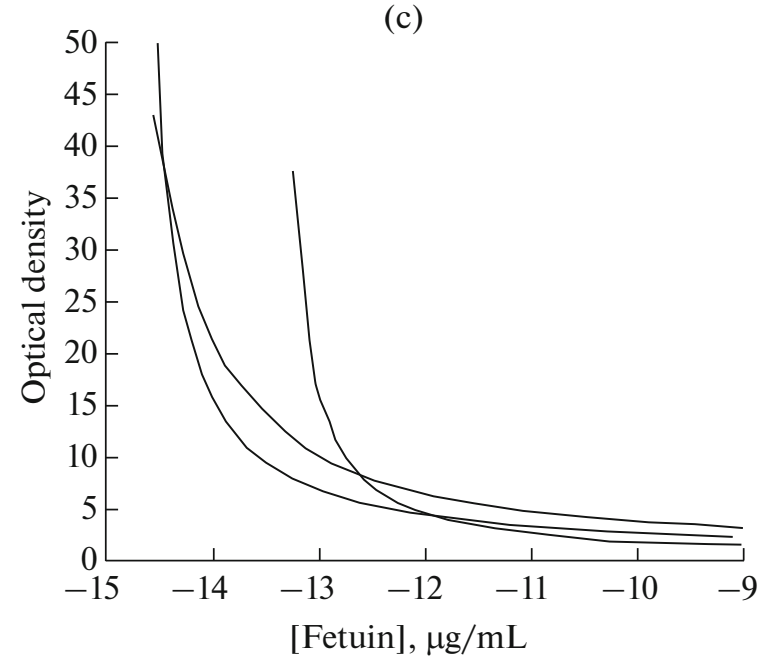

(b)

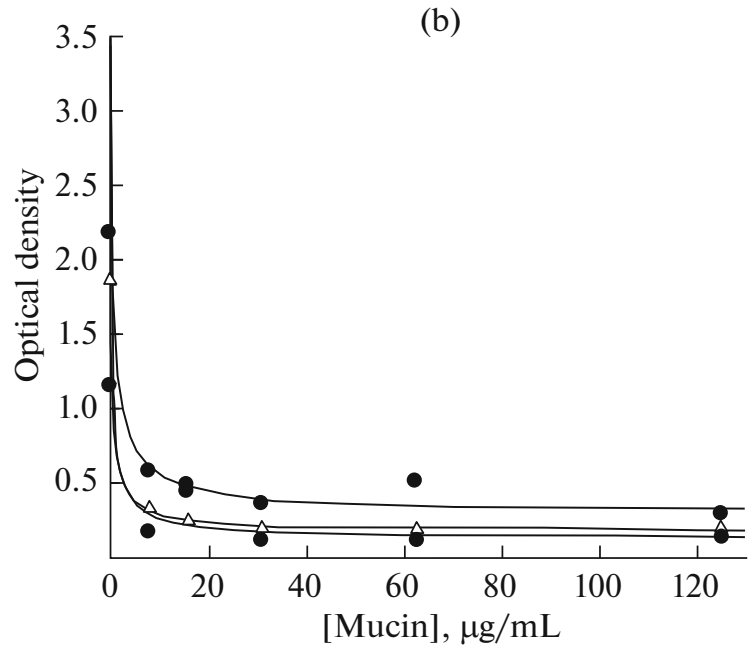

(d)

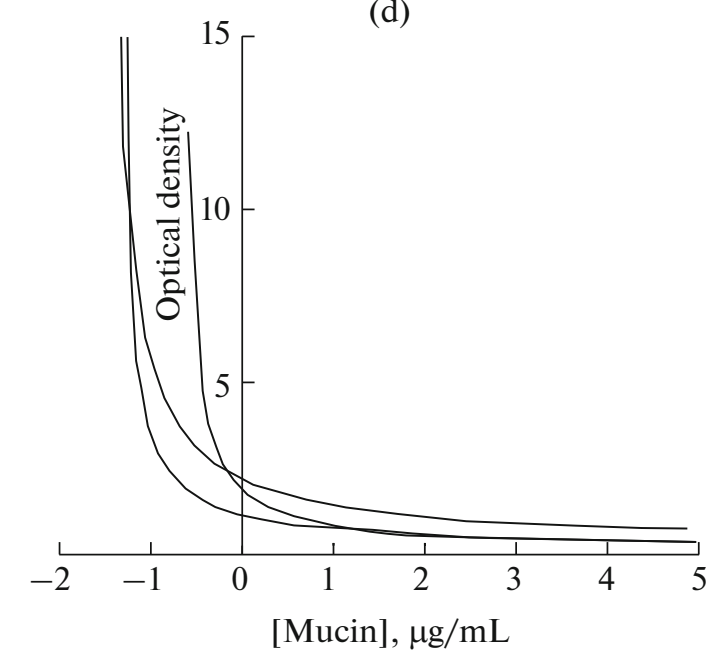

Fig. 1. The dependence of the optical density on the concentration of the competitive fetuin (a) or mucin (b) protein. The three extrusion curves correspond to three different dilutions of the biotinylated protein for the influenza A/California/7/2009 (H1N1pdm09) strain. The graphs (c) and (d) show enlarged corresponding dependencies.

(a)

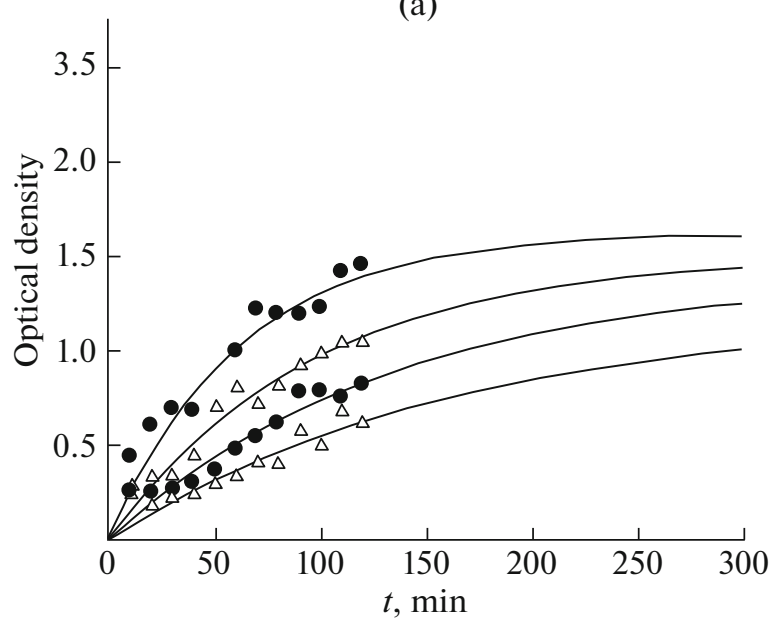

(b)

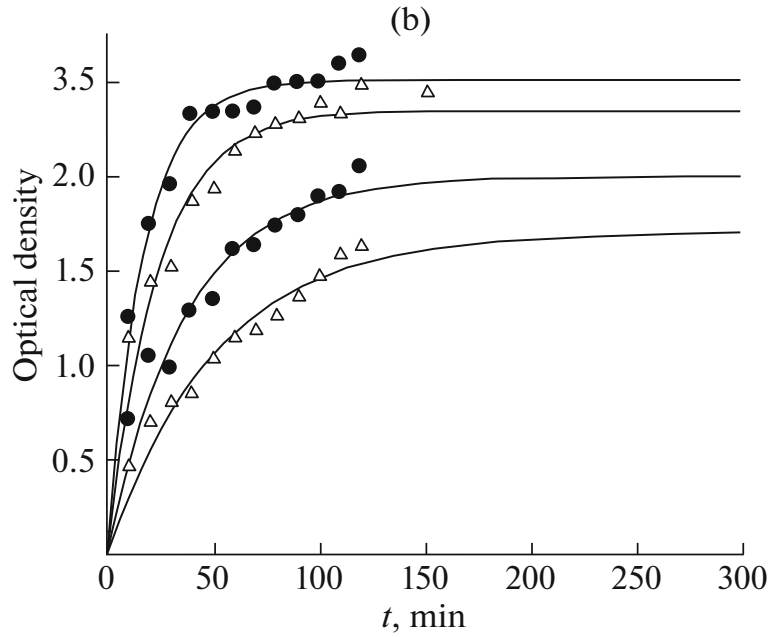

Fig. 2. The kinetics of binding of the influenza A/California/7/2009 (H1N1pdm09) strain to fetuin (a) and mucin (b). Four curves correspond to four different dilutions of biotinylated protein. 


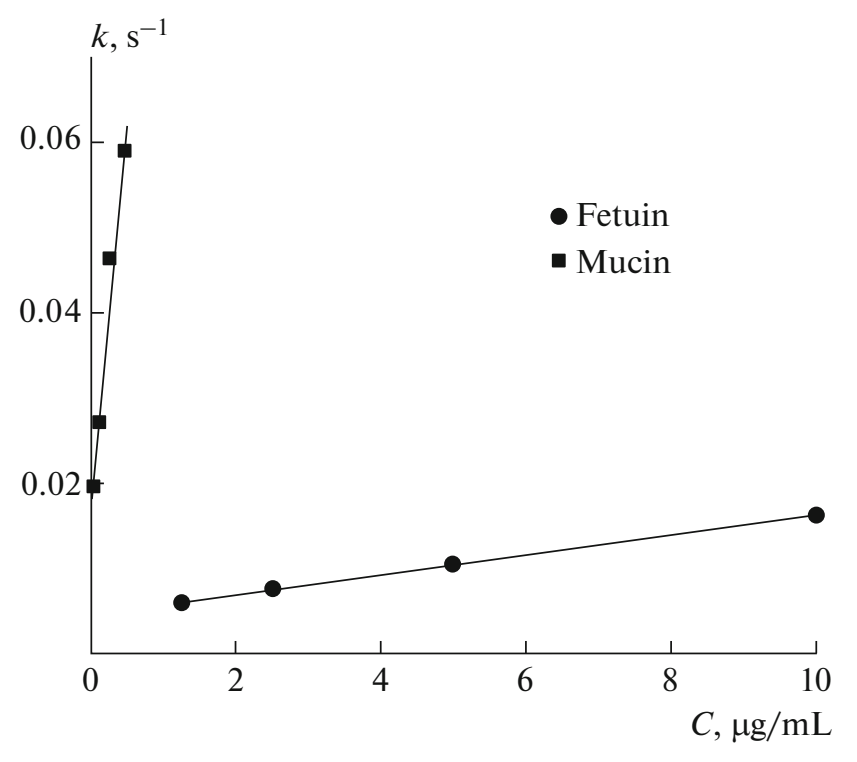

Fig. 3. The dependences of the calculated $k$ parameters on the concentrations of fetuin and mucin for the influenza A/California/7/2009 (H1N1pdm09) strain.

virus and two proteins, calculated by three methods: Dixon plots in direct coordinates, the method using the half-maximal inhibitory concentration $\left(I C_{50}\right)$, and the kinetic method.

\section{DISCUSSION}

The values of the affinity constant of influenza A/California/7/2009 (H1N1pdm09) virus for fetuin (equal to the inverse values of the equilibrium dissociation constant), measured by Dixon plot analysis in direct coordinates and by the $I C_{50}$ method, differ on average by no more than $1.5 \mu \mathrm{mol}^{-1}$. Measurements by the kinetic method show greater affinity in comparison with competitive methods: the average difference is $6.8-8.3 \mu \mathrm{mol}^{-1}$. The value of the affinity of the virus for mucin, as measured by the kinetic method, also exceeds the values obtained by competitive methods. However, the difference between the kinetic and competitive methods (in comparison with fetuin) is more significant: on average, it is $513.2-574.8 \mu \mathrm{mol}^{-1}$. Such a difference between the competitive and kinetic methods is explained by the effect of unspecific binding of competing proteins during a competitive measurement in ELISA, which underestimates the real value of the dissociation constant. These results indicate that the effect of unspecific binding of competing proteins on the result is significant for mucin and moderate for fetuin.

\section{CONCLUSIONS}

Commonly used competitive ELISA methods for measuring the affinity of the influenza virus are relatively adequate for assays with fetuin, but may significantly distort the result when mucin is used. The kinetic method tested during this study has an advantage over competitive methods in the case of a problem of unspecific binding for some proteins.

Thus, the kinetic method can be used to measure the receptor specificity of influenza A viruses along with competitive methods in ELISA and other existing methods. Moreover, it is the best ELISA method for measuring the binding affinity of a virus for proteins that have a natural ability to bind to the protein of their kind, which was shown during this study using mucin as an example.

\section{COMPLIANCE WITH ETHICAL STANDARDS}

The authors declare that they have no conflict of interest. This article does not contain any studies involving animals or human participants performed by any of the authors.

Table 1. The equilibrium dissociation constants for the influenza A/California/7/2009 (H1N1pdm09) strain with fetuin and mucin

\begin{tabular}{l|c|c}
\hline \multirow{2}{*}{ Method } & \multicolumn{2}{|c}{$K_{\mathrm{d}}, \mathrm{nM}$} \\
\cline { 2 - 3 } & fetuin & mucin \\
\hline $\begin{array}{l}\text { Dixon plots in direct coordinates } \\
\text { Method using half-maximal inhibitory } \\
\text { concentration }\left(I C_{50}\right)\end{array}$ & $272.9 \pm 12.2$ & $12.2 \pm 5.9$ \\
Kinetic method & $192.9 \pm 43.7$ & $49.1 \pm 2.3$ \\
\hline
\end{tabular}




\section{REFERENCES}

1. T. N. Il'icheva, S. V. Netesov, and V. N. Gureev, Influenza Viruses: Methodological Textbook (Novosibirsk State Univ., Novosibirsk, 2014).Chap. 1 [in Russian].

2. A. S. Gambaryan, S. S. Iamnikova, D. K. L'vov, et al., Mol. Biol. 36, 542 (2002).

3. K. Shinya, M. Ebina, S. Yamada, et al., Nature 440, 435 (2006).

4. R. McBride, J. C. Paulson, and R. P. de Vries, J. Vis. Exp., No 111, e53847, doi (2016). https://doi.org/10.3791/53847

5. S. D. Varfolomeev and K. G. Gurevich, Biokinetics: $A$ Practical Course (FAIR-Press, Moscow, 1999) [in Russian].

6. S. D. Varfolomeev and S. V. Zaitsev, Kinetic Methods in Biochemical Research (Moscow State Univ., Moscow, 1982) [in Russian].
7. T. R. Maines, A. Jayaraman, J. A. Belser, et al., Science 325, 484 (2009).

8. R. Xu, R. McBride, C. M. Nycholat, et al., J. Virol. 86, 982 (2012).

9. R. A. Childs, A. S. Palma, S. Wharton, et al., Nat. Biotechnol. 27, 797 (2009).

10. J. U. Baenziger and D. Fiete, J. Biol. Chem. 254 (3), 789 (1979).

11. S. Mizan, A. Henk, A. Stallings, et al., J. Bacteriol. 182, 6874 (2000).

12. H. Schachter, E. J. McGuire, and S. Roseman, J. Biol. Chem. 246, 5321 (1971).

13. S. C. Baos, D. B. Phillips, L. Wildling, et al., Biophys. J. 102, 176 (2012).

14. M. C. Rose and J. A. Voynow, Physiol. Rev. 86 (1), 245 (2006).

Translated by A. Panyushkina 